\title{
Proceso de Bolonia (III). Educación en valores: profesionalismo
}

\author{
Arcadi Gual, Jordi Palés-Argullós, María Nolla-Domenjó, Albert Oriol-Bosch
}

\begin{abstract}
I will highlight the medical school as a moral community and training as a process of moral enculturation ... and how issues of professionalism may be better addressed within medical training ...
\end{abstract}

F.C. Hafferty [37]

El desarrollo profesional y personal de los estudiantes de grado y de formación especializada como objetivo de la educación médica requiere disponer de definiciones normativas sobre el contenido y los contextos de los términos 'profesional', 'profesionalidad' y 'profesionalismo' para que el sistema educativo pueda diseñar estrategias educativas y evaluadoras eficientes [1]. Esta necesidad no surge por carencias reales, puesto que las reflexiones abundan en la bibliografía de la última década del siglo pasado [2-6] y de la primera del presente [7-14], sino más bien por exceso y porque los diferentes grupos utilizan el término 'profesionalismo' de manera y con propósitos distintos. Este término se ha mostrado fácilmente identificable pero difícilmente definible [1]. Por esta razón, la Federación Mundial de Educación Médica (WFME) ha constituido un grupo de trabajo que actualmente está en ello (WFME Task Force 2010).

El grupo de trabajo de la WFME podrá disponer, para el desarrollo de su tarea, del trabajo realizado anteriormente por otras instituciones. Entre estas sobresale, en Gran Bretaña, el General Medical Council (GMC), institución con capacidades normativas bien definidas que ha producido dos documentos: en 2009, la última versión de Tomorrow's Doctors [15], y en 2006, Good Medical Practice [16], donde se describen con detalle los atributos y los comportamientos esperados en los miembros de la profesión médica; en Norteamérica, destaca en 2007 el Accreditation Council for Graduate Medical Education (ACGME) con su Outcome Project [17]; en 2002, el American Board of Internal Medicine (ABIM) con su Project Professionalism, que culminó con el documento The Physician Charter [18]; en 1998, la American Association of Medical Schools (AAMC) con su proyecto de objetivos de aprendizaje AAMC [19], y en 2005, el Royal College of Physicians and Surgeons of Canada, con el documento conocido por la abreviatura CanMeds (RCPSC 2005) [20], y la Association of Faculties of Medicine of Canada (AFMC), con el documento The Future of Medical Education in Canada [21]. En España, recientemente, en 2010, el Consejo General de Colegios de Médicos (CGCOM) y la Organización Médica Colegial (OMC) han formulado las definiciones de 'profesional' y 'profesionalismo médico' [22] (Tabla I).

El sistema educativo no está falto de las referencias normativas necesarias [1], aunque quizá sus miembros no hayan internalizado aún que el proceso del que son responsables es mucho más que una transferencia de conocimientos asociada a un entrenamiento para adquirir habilidades. Siendo estos factores (conocimientos y habilidades) imprescindibles, el proceso de formación de un médico requiere, además, un proceso transformacional del sujeto que aprende, por el cual éste llega no tan sólo a saber y a saber hacer más, sino también a ser distinto e incorporarse a una comunidad profesional.

Para muchos, estas consideraciones son de eruditos y están lejos de las necesidades de la praxis profesional cotidiana. Borrell et al [8] ponen ejemplos cotidianos (Tabla II) que evidencian que la profesionalidad está permanentemente en juego en la actuación y en el quehacer médico de cada día, distinguiendo entre el quehacer técnico del médico y el del médico que actúa como profesional, ya que este último 'aplica el saber y las técnicas ... a partir de un juicio informado por la virtud' (sic). Este 'jui-
Fundación Educación Médica (A. Gual, J. Palés-Argullós, M. NollaDomenjó, A. Oriol-Bosch). Facultad de Medicina; Universitat de Barcelona (A. Gual, J. Palés-Argullós). Fundación Doctor Robert; Universitat Autònoma de Barcelona (M. Nolla-Domenjó). Barcelona, España.

Correspondencia:

Dr. Jordi Palés Argullós. Departamento de Ciencias Fisiológicas I. Facultad de Medicina. Universitat de Barcelona. Casanova, 143. E-08036 Barcelona. E-mail: jpales@ub.edu

(c) 2011 Educación Médica 
Tabla I. Definiciones de profesión médica, profesional médico y profesionalismo médico [22].

Profesión médica

Ocupación basada en el desempeño de tareas encaminadas a promover y restablecer la salud y a identificar, diagnosticar y curar enfermedades aplicando un cuerpo de conocimiento especializado propio de nivel superior, en la que preside el espíritu de servicio y en la que se persigue el beneficio del paciente antes que el propio, y para la cual se requiere que las partes garanticen:

- La producción, el uso y la transmisión del conocimiento científico.

- La mejora permanente para prestar la mejor atención posible.

- La aplicación del conocimiento de forma ética y competente.

- Que la práctica profesional se oriente hacia las necesidades de salud de las personas y la comunidad.

Profesional médico

Médico o médica titulado/a comprometido/a con los principios éticos y deontológicos y los valores de la profesión médica y cuya conducta se ciñe a dichos principios y valores

Profesionalismo médico

Conjunto de principios éticos y deontológicos, valores y conductas que sustentan compromisos de los profesionales de la medicina con el servicio a los ciudadanos, que evolucionan con los cambios sociales, y que avalan la confianza que la población tiene en los médicos.

Principios fundamentales del profesionalismo médico

El ejercicio de la profesión médica exige anteponer los intereses del paciente a los del propio médico, base de la confianza que el paciente deposita en el médico, una exigencia que se sustenta, entre otros, por los principios de beneficencia, no maleficencia, autonomía y justicia.

Valores fundamentales de la profesión médica

Los profesionales de la medicina ponen a disposición de la población los conocimientos, las habilidades y el buen juicio para promover y restablecer la salud, prevenir y proteger de la enfermedad, y mantener y mejorar el bienestar de los ciudadanos. En consecuencia, la práctica diaria del profesional médico implica el compromiso con:

- La integridad en la utilización del conocimiento y en la optimización de los recursos.

- La compasión como guía de acción frente al sufrimiento.

- La mejora permanente en el desempeño profesional para garantizar la mejor asistencia posible al ciudadano.

- La colaboración con todos los profesionales e instituciones sanitarias en aras de la mejora de la salud y del bienestar de la población.

cio informado por la virtud' es algo más que la aplicación de un código ético o de buena praxis estándar, puesto que exige la reflexión personal sobre las propias reacciones emocionales, las influencias de nuestro entorno (cultura institucional) y los prejuicios de las costumbres y creencias. La necesaria formación en valores exige por tanto algo más que el conocimiento de los códigos éticos y de buena praxis (conocimiento formal), debiendo incorporar el desarrollo del recto juicio para su aplicación contextualizada (conocimiento experiencial).

La formación necesaria para llegar a ser médico es un proceso de aculturación o socialización secundaria por la que quien aprende adopta la normativa de la profesión y su credo profesional (profesionalismo) para expresarlo en forma de conductas aceptables (profesionalidad) de la profesión médica. Aprender a ser médico consiste en aprender a ajustarse a las normas de la profesión, lo que ocurre necesariamente en contextos sociales determinados (clínicos) e incluye las reacciones de los otros miembros del entorno, especialmente los influyentes [23].
El aprendizaje se lleva a cabo a través del modelaje que genera la conducta de los otros miembros del contexto clínico o educativo, especialmente observando el impacto social de la conducta de los más relevantes [24], un aprendizaje difícil que ocurre entre conflictos normativos y aprendiendo a controlar las emociones propias (miedo, disgusto, desconcierto, etc.) [14]. Los conflictos normativos exigen equilibrios y la gestión de tensiones de rol. Ello se ha puesto de manifiesto en patrones cíclicos en la empatía, el razonamiento moral, el humanismo o el cinismo de los que están aprendiendo [25].

Los alumnos admitidos en las facultades de medicina llegan con un potente e ingenuo espíritu de servicio altamente idealizado que sufre una erosión a medida que progresa su formación, hecho que produjo una alarma notable al detectarse [26]. El proceso vuelve a repetirse a lo largo de la residencia, pero en general, el resultado final de lo que algunos han interpretado como un proceso de deterioro moral a lo largo de la formación no es la existencia de un cuerpo profesional envilecido, más bien 
al contrario, pues son excepción los fallos flagrantes y graves de profesionalismo. Lo que se ha detectado son las consecuencias de los ajustes en la aplicación de unos principios idealizados y teóricos a la complejidad de los contextos reales, a los tanteos en el aprender a resolver dilemas éticos y a las respuestas emocionales defensivas a las tensiones que la realidad crea en los neófitos que aprenden. Se trata pues de procesos cíclicos mediante los cuales van progresando en su transformación hacia un devenir profesional [27].

Sin embargo, el entorno clínico no siempre constituye un clima ideal para el aprendizaje. Pueden parecer justificables en ciertos entornos algunas decisiones poco éticas por deferencia a la autoridad o lealtad [28], lo que acostumbra a acompañarse del uso de 'humor negro' y del etiquetaje del paciente, deshumanizándolo.

Puede tratarse de defensas mentales que se acompañan de desorientación moral de los que aprenden, aunque en realidad pueden ser sólo un proceso de adaptación a las tensiones frente a la realidad y representar fallos que se producen en todo aprendizaje. Estos hechos sólo deberían constituir una preocupación cuando su prevalencia sea elevada o se incardinen en el contexto clínico actitudes de excesivo distanciamiento con los pacientes hasta desentenderse de ellos [24].

El aprendizaje y la adaptación normativa es social y depende de las oportunidades para captar las señales emitidas por los otros, especialmente los más influyentes, con respecto a las conductas esperadas [29] y del balance entre las oportunidades de actuación de acuerdo o en contraposición a dichas expectativas [30], lo cual depende de las expectativas acerca del riesgo de ser castigados [31].

Las oportunidades mencionadas dependen del grado de 'observabilidad' de las conductas y de la 'dispersabilidad' de dicha información en la red social, lo que a su vez depende de sus características. Una red social 'densa' permite que la reputación actúe controlando las conductas, en tanto que una red 'laxa' no facilita la acción punitiva del conjunto de los miembros de la red. Las redes sociales definen importantes dimensiones del entorno normativo a las que se hallan expuestos los que aprenden e influyen en la forma en que reaccionan a los fallos normativos.

Las redes sociales de los médicos incluyen, además de los colegas, a otros profesionales sanitarios y a los empleadores, gestores, financiadores y reguladores además de, naturalmente, a los pacientes, cada uno de los cuales posee expectativas específicas sobre las conductas esperadas, lo que hace que
Tabla II. Retos cotidianos a la profesionalidad [8].

¿Cómo gestionar el escaso tiempo disponible para la atención de cada paciente?

¿Cuándo debo dar por acabada una entrevista, aunque el paciente desee proseguirla?

¿Qué hacer cuando se está tan cansado que no sea prudente realizar un acto quirúrgico?

¿Cómo mostrarse seguro de las recomendaciones sin llegar a ser coactivo?

¿Hasta qué punto debe facilitarse el acceso a cierto grado de invalidez si no se tiene la seguridad de que el paciente sea acreedor a este?

Si no se tiene certeza del diagnóstico, ¿debería saberlo el paciente?

¿Hasta qué punto es necesario preocuparse por un paciente que no se preocupa por su salud?

¿Es preciso informar sobre el maltrato a pacientes por un colega o sobre su posible alcoholismo?

el proceso de aprendizaje y adaptación a la normativa no sea precisamente un proceso sencillo. El contacto inadecuado o insuficiente con todo este contingente de recursos humamos, y muy especialmente con el personal de enfermería, puede contribuir a un clima de aprendizaje pobre [33] y a una adaptación y búsqueda de consejo insuficientes.

La centralidad en la red social es un componente significativo. La marginalidad genera aislamiento y es un factor importante en los ajustes inadecuados a la normativa profesional. Quienes aprenden acostumbran a ser ubicados en la periferia de las redes sociales clínicas con una rígida jerarquización, lo que no facilita la comunicación con los supervisores [32]. Los repetidos fallos de profesionalismo en los comportamientos pueden, a su vez, conducir a la marginación en la red social aislando al 'culpable' por las actitudes de los otros miembros de la red o porque el sujeto busque posiciones con menor visibilidad.

$\mathrm{El}$ ajuste individual a la normativa profesional es, pues, inseparable del contexto social y de las reacciones de los demás, especialmente de los más influyentes. El grado de ajuste a la normativa profesional se correlaciona con otros elementos que no han sido objeto de consideración hasta el momento, como las interacciones entre las características individuales del que aprende, el clima de aprendizaje del contexto clínico en que se produce el aprendizaje y las influencias de las redes sociales sobre las conductas éticas en dichas organizaciones [33] (Tabla III).

Los ajustes individuales y grupales a la norma están relacionados y se conforman recíprocamente, pero los efectos sobre la actuación individual dependen de las estrategias que emplea cada uno [34]. 
Tabla III. Correlación de constructos psicosociales con el ajuste individual a las normas profesionales [23].

\begin{tabular}{lc}
\hline Constructos & $\begin{array}{c}\text { Dirección de } \\
\text { la asociación }\end{array}$ \\
\hline $\begin{array}{l}\text { Posición en la red social } \\
\text { Fuerza y reciprocidad del vínculo } \\
\text { Centralidad }\end{array}$ & + \\
\hline Características y circunstancias individuales & + \\
Empatía & + \\
Desarrollo cognitivo moral & + \\
Habilidades comunicativas & + \\
Experiencia clínica & + \\
Nuevo entorno cultural & - \\
Normativas nuevas & - \\
Fatiga & - \\
\hline
\end{tabular}

Características de la organización

Fallos de profesionalidad visibles

Sistemas de reconocimiento y refuerzo de conductas positivas

Estructura normativa

Cultura profesional y

códigos de conducta consolidados

Roles claramente establecidos

Congruencia normativa

Características de la red social

Densidad

$+$

Presencia de 'cliques' y agujeros estructurales

Sin embargo, las normas profesionales se adquieren a través de mecanismos de aprendizaje social y cualquier característica personal que interfiera dicho aprendizaje se correlacionará negativamente con el ajuste a la norma. Se ha descrito ya cómo deficiencias en elementos como la empatía [35] o el desarrollo cognitivo moral [33] se correlacionan con el ajuste normativo al afectar la habilidad individual para el aprendizaje social.

Algunas características de la cultura de la organización permiten predecir su efectividad educativa. Un gran consenso normativo produce un feedback informal consistente, menos mensajes contra- dictorios y menor exposición a conductas inapropiadas [31]. Donde mayor sea la coherencia con la norma es donde se dará una mayor densidad y una mayor centralidad en la red social [33]. Por ello deben ser objeto de interés de la educación médica tanto la cultura institucional como las características de sus redes sociales.

A nivel individual, Reiser [36] señala la importancia de lo que el docente hace, y cita el comienzo de $E l$ médico de Hipócrates: 'La dignidad del médico requiere que ... lleve una vida regulada ... y que su carácter sea caballeroso ... y sea afable con todos', indicando la gran importancia del rol de modelaje del médico-maestro, que no se limita a lo que sabe y enseña y a lo que como médico hace y enseña a hacer, sino que incluye también qué es lo que hace como persona y cómo a través de su conducta muestra cuáles son sus valores. Lo que el médico hace fuera de su actuación como médico anticipa lo que es en su calidad de tal. Los estudiantes aprenden a confiar en sus profesores no tanto por los valores que proclaman como por cómo ven que los ejercen. Los maestros influyen en los que aprenden por el interés y el respeto que les muestran hacia el esfuerzo que realizan para aprender y desarrollarse. El juramento hipocrático, creador del concepto de profesión médica occidental, desarrolla también la bidireccionalidad de los compromisos entre maestros y los que aprenden, colocando la reciprocidad ética en el centro de la relación. Esta relación maestro-estudiante prefigura la relación estudiante-paciente, el uso de la autoridad del que tiene conocimiento sobre el que no lo tiene. Los educadores deben ayudar a los que aprenden a entender la complejidad de sus relaciones, al tiempo que no deben considerarlos como contenedores pasivos a los que tan sólo deben llenar de conocimientos. A su vez, quienes aprenden tienen compromisos recíprocos de alto contenido ético que se tratarán en otro lugar. Baste con tener en cuenta ahora que el ignorar los preceptos éticos del aprender puede dificultar el devenir de un estudiante en un profesional ético o, sencillamente, en un profesional, puesto que sin ética no es posible ser un profesional.

El orden moral de una facultad de medicina se construye sobre las relaciones entre estudiantes y docentes, pero también sobre las políticas institucionales que configuran su cultura [36] y que alinea sus recursos y personal para alcanzar unos fines determinados con un estilo concreto. Estas políticas institucionales se explicitan en lo que se conoce como 'misión' institucional y en los códigos de conducta que promulga, y en aquellas formas de hacer que reconoce como buenas o aquellas otras que, no 
siéndolo, se toleran tácitamente. Este orden moral que prevalece en una institución habla con voz alta de lo que es y en lo que cree dicha institución. La fuerza interior de una facultad de medicina se sostiene por su capacidad para generar confianzas recíprocas, preocupación por la dignidad de las personas y su consentimiento, por su compromiso con el debate abierto sobre las opciones institucionales y el reconocimiento de que son las acciones y las omisiones, como los silencios, los que crean responsabilidades y evidencian sus propósitos. Ha llegado pues la hora de revisar las 'misiones' de las facultades de medicina para comprobar su vigencia y actualizarlas, así como reflexionar sobre las características de su cultura observando su actuación.

Hafferty [37] plantea la molesta cuestión: ¿dónde se ubican en el proceso de la formación de médicos las actividades pedagógicas diseñadas para culturalizarlos en valores y orientarlos normativamente? En su búsqueda entre las 126 facultades de medicina de Estados Unidos encontró sólo una, la de Universidad de Wisconsin, que explicitara en su currículo un curso dedicado al tema del profesionalismo -'Profession of Medicine Program' [38]-, y otras cuatro con cursos de los que se podía inferir que lo trataban más o menos directamente. Consultadas dichas facultades para que aportaran evidencias de los resultados obtenidos, las respuestas fueron esquivas, sin aportar resultados de medidas evaluadoras. Sin embargo, la situación ha cambiado en esta última década, apareciendo facultades plenamente comprometidas con la orientación a los valores y el desarrollo del profesionalismo [39]. A comienzos de la segunda década del siglo XXI parece legítimo preguntar en qué situación nos encontramos después de un proceso de adaptación al proceso de Bolonia, cuando las facultades españolas han recibido ya la autorización ministerial para desarrollar los nuevos estudios de grado tras la validación de la ANECA (Agencia Nacional de Evaluación de la Calidad y Acreditación).

La formación en profesionalismo debe atender los tres ámbitos de toda formación:

- Contenidos o bases cognitivas.

- Pedagogías o estrategias para enseñar y aprender.

- Evaluación para saber hasta qué punto los resultados obtenidos se acercan a los objetivos deseados.

\section{Bases cognitivas del profesionalismo}

El médico deberá saber lo que es el profesionalismo y poder explicarlo [40], lo que incluye las raíces históricas de las profesiones y de la medicina como profesión, las definiciones normativas, los atributos esperados del profesional médico (sanador) y las expectativas cambiantes de la sociedad al respecto, el contrato social entre la medicina y la sociedad, y los compromisos que comporta la creciente complejidad del entorno asistencial y el papel del médico en los equipos asistenciales.

En estas bases cognitivas, la ética (valores), la sociología (relaciones), la psicología (conductas) y la antropología (cultura) deben aportan sus perspectivas.

\section{Pedagogías para aprender y enseñar profesionalismo}

La teoría del 'aprendizaje situacional' parece ser la más adecuada para articular la formación de las profesiones, por tratarse éstas de 'comunidades o culturas unidas en redes sociales estructuradas sobre creencias' [41]. En la teoría del aprendizaje situacional se aúna la base cognitiva con el aprendizaje experiencial, es decir, el 'saber el qué' con el 'saber el cómo' introduciendo el aprendizaje en las actividades auténticas, transformando el conocimiento abstracto y teórico en conocimiento útil y utilizable [42]. El aprendizaje situacional se basa en la noción de que el conocimiento es situacional y está influido por la actividad, la cultura y el contexto en que se ha generado [43]. El aprendizaje cognitivo, colaborativo, por la práctica, con reflexión y desarrollando las habilidades para aprender son los elementos de la teoría del aprendizaje situacional, que en medicina se han producido desde siempre en contextos clínicos, y que se lleva a cabo en cuatro fases: la de modelaje, la de andamiaje (de apoyo), la de responsabilización y la del coaching [44].

Para promover el aprendizaje colaborativo sirve la solución colegiada de problemas, los juegos de roles y la confrontación a estrategias equivocadas o a errores conceptuales, siempre trabajando en grupos pequeños para que todos puedan tener una papel activo que contribuya al desarrollo de las habilidades colaborativas.

La reflexión, elemento nuclear en la teoría del aprendizaje situacional, fue identificada ya por Schön [45] como una competencia profesional esencial, en tanto que la práctica repetitiva sirve para desarrollar automatismos necesarios, siendo otro elemento esencial del aprendizaje experiencial.

Según la teoría del aprendizaje situacional, el papel del docente es múltiple y va más allá del de experto en contenidos formales para asumir el rol de modelo y compartir con sus alumnos el rol de ense- 
Tabla IV. Evaluación del profesionalismo [54]

Desarrollar un plan institucional para la evaluación del profesionalismo; enfoques desarrollados por grupos aislados fracasarán

Acordar lo que significa profesionalismo para la institución con la participación de todos los agentes implicados

Seleccionar las conductas sobre las que se centrará el proceso

Decidir si la evaluación va a ser sumativa o formativa; en caso de que se vayan a realizar ambas, deberá quedar claro cuáles son sumativas y cuáles formativas

Identificar los instrumentos para medir las conductas teniendo en cuenta:

El contexto, el conflicto y la resolución

La transparencia y la simetría del proceso

El uso de múltiples evaluadores y

tipos de medida para potenciar la validez

Formar a los evaluadores

Implementar el programa y evaluarlo

ñar (aprendizaje colaborativo). La teoría del aprendizaje situacional va de la mano del aprendizaje experiencial ('aprender haciendo'), un proceso de participación en el trabajo del equipo asistencial, por el cual el principiante se inicia en la marginalidad del grupo y, a medida que incrementa su capacidad de contribuir a las tareas, progresa hacia la centralidad, donde los expertos realizan las tareas más complejas.

Los principios del aprendizaje de adultos deben tenerse en cuenta en el diseño educativo, especialmente en la selección de las estrategias y los métodos (juegos de rol, simulaciones, discusión de pequeños grupos y, sobre todo, modelaje de roles). El role modeling, o modelaje de roles, es la mejor metodología para mostrar e instilar actitudes, conductas y valores profesionales a los estudiantes y residentes [46], lo que lamentablemente se olvida a menudo y, lo que es peor, se ejerce pobremente [47]. El modelaje de rol exige que quien actúa de modelo sea clínicamente competente (conocimientos, habilidades clínicas y comunicativas, buen juicio y capacidad para decidir), un habilidoso educador (comunicador, retroalimentador y creador de oportunidades para la reflexión) y esté dotado de cualidades personales (honestidad, compasión, integridad, entusiasmo y compromiso con la excelencia). El uso de casos, reales o en viñetas, es otro instrumento poderoso para la formación en profesionalismo [48]. El uso del portafolio [49], del arte [50], del vídeo y de la narrativa [51] muestran su utilidad en contextos educativos innovadores.

El contexto clínico en el que se aprende con frecuencia es 'tóxico', dificultando la enseñanza y el aprendizaje [52]. Merece la pena que las facultades analicen su clima educativo, que depende de su cultura institucional, pues sin climas educativos adecuados, que conforman los currículos ocultos [53], difícilmente se logrará alcanzar los objetivos educativos deseados.

\section{Evaluación del profesionalismo}

La evaluación del profesionalismo debe cumplir con las mismas exigencias de cualquier evaluación, aunque hay que aceptar que en principio se plantean dificultades importantes. Stern [54] propone siete pasos para guiar la implementación de la evaluación del profesionalismo (Tabla IV).

La facultad capaz de implementar con consenso institucional un sistema de evaluación del profesionalismo encontrará en los resultados obtenidos la información para diseñar las estrategias de mejora y la movilización de sus energías internas para hacerlo.

Hafferty [37] comenta que, a través de las generaciones, los estudiantes se plantean entre ellos el dilema retórico siguiente: '¿A quién preferirías como médico personal, uno con mucho conocimiento pero insensible o uno afectuoso pero con poco conocimiento?'. Es un debate sobre las dos almas de la medicina, la científico-técnica y la del sanador, la que se centra en enfermedades y la que atiende a pacientes. El profesionalismo resuelve este dilema, puesto que la búsqueda de la excelencia en lo científicotécnico y en el desarrollo de los atributos de servicio al paciente son inseparables de la profesión y deben desarrollarse en paralelo. El dilema desaparece si sustituimos la emotividad, que es sólo una parte importante del profesionalismo, por los motivos y los principios que deben regir al médico. A la hora de escoger en quién confiaría, ningún estudiante tendrá dudas si se trata del Dr. Frankenstein o del Dr. Moreau, descritos respectivamente en las novelas de M. Shelley y H.G. Wells en el mundo de ficción, o si se trata del Dr. Harold Shipman o del Dr. Juan Maeso en el mundo real. El profesionalismo debe entenderse como el conjunto de obligaciones y compromisos libremente asumidos por aquellos que desean pertenecer a un grupo profesional y no se contentan con ser técnicos de alta cualificación.

Los principios que rigen las actuaciones de la profesión y los compromisos que ésta adquiere fren- 
te a la sociedad deben ser conocidos formalmente y plenamente internalizados por los estudiantes en su devenir a médicos. Los principios no pueden sino derivarse de los de la ética general y los compromisos han sido recogidos de forma actualizada para las realidades actuales (Tabla V).

\section{Conclusiones}

- Son muchas las referencias normativas acerca del profesionalismo en particular y la formación en valores en general, pero los estamentos docentes, ya sean de grado o de formación especializada, y los estamentos profesionales, colegios profesionales o sociedades científicas distan de asumirlos en sus programas docentes o en su ideario o misión institucional.

- Es difícil pensar que un alumno instruido con todos los conocimientos y todas las habilidades necesarias para ser un médico especialista, pero que no haya convivido nunca en los espacios propios de una facultad de medicina y de un hospital o centro de primaria, pudiera enfrentarse a la práctica, porque a pesar de disponer de los imprescindibles conocimientos y habilidades, carecería del proceso transformacional que le permite incorporarse a la comunidad profesional de los médicos que los distingue de los técnicos de alta cualificación.

- La necesaria formación en valores exige algo más que el conocimiento de los códigos éticos y de buena praxis (conocimiento formal), ya que debe incorporar el desarrollo del recto juicio para su aplicación contextualizada (conocimiento experiencial).

- La formación necesaria para llegar a ser médico es un proceso de aculturación o socialización secundaria por la que quien aprende adopta la normativa de la profesión y su credo profesional (profesionalismo) para expresarlo en forma de conductas aceptables (profesionalidad) de la profesión médica. Este aprendizaje se lleva a cabo a través del modelaje que genera la conducta de los otros miembros del contexto clínico o educativo.

- El entorno clínico, generador del currículo oculto, no siempre constituye un clima ideal para el aprendizaje. Repetidamente, el discurso que pone al paciente como centro de la actividad del médico no acompaña las conductas observables en las diferentes instituciones sanitarias. Son pues estas conductas observables, y no el discurso, las que deben ser objeto de principal atención y mejora por parte de los docentes, los gestores y todo
Tabla V. Principios y responsabilidades de la profesión médica [18].

Principios

Primacía del bienestar del paciente

Autonomía del paciente

Justicia social

Responsabilidades

Compromiso con la competencia profesional

Compromiso con la honestidad con los pacientes

Compromiso con la confidencialidad del paciente

Compromiso con el establecimiento de relaciones adecuadas con los pacientes

Compromiso con una atención sanitaria de mayor calidad

Compromiso con la mejora del acceso a la asistencia médica

Compromiso con la distribución justa de los recursos finitos

Compromiso con el saber científico

Compromiso con el mantenimiento de una confianza sólida gracias a la solución de los conflictos de interés

Compromiso con las responsabilidades profesionales

el personal de la institución, y si cabe de forma muy particular, de cada médico que crea y quiera ser un profesional.

- Las redes sociales -los médicos están y actúan inmersos en una red social- definen importantes dimensiones del entorno normativo a las que se hallan expuestos los que aprenden e influyen en la forma en que reaccionan a los fallos normativos. La red social de los médicos es altamente compleja por el gran y diferente número de actores con distintas expectativas y, además, porque como en todas las redes sociales, quienes aprenden son ubicados en la periferia con una rígida jerarquización que no facilita la comunicación con los supervisores. Si la red es compacta, 'densa', permite que la reputación actúe controlando las conductas, en tanto que una red 'laxa' no facilita la acción punitiva del conjunto de los miembros de la red.

- Deben ser objeto de interés de la educación médica tanto la cultura institucional como las características de sus redes sociales, ya que la cultura de la organización permite predecir su efectividad educativa. Los estudiantes aprenden a confiar en sus profesores/tutores no tanto por los valores que proclaman como por cómo ven que 
los ejercen. La relación maestro-estudiante prefigura la relación estudiante-paciente, el uso de la autoridad del que tiene conocimiento sobre el que no lo tiene. El ignorar los preceptos éticos del aprender puede dificultar el devenir de un estudiante en un profesional ético o en un profesional, puesto que sin ética no es posible ser un profesional.

- El orden moral de una facultad de medicina se construye sobre las relaciones entre estudiantes y docentes, pero también sobre las políticas institucionales ('misión' institucional) que configuran su cultura y que alinea sus recursos y personal para alcanzar unos fines determinados con un estilo específico. El orden moral que prevalece en una institución define lo que es y en lo que cree una institución. Por ello, las instituciones docentes deben revisar tanto las 'misiones' como las características de su cultura institucional para comprobar la vigencia de las primeras y la reflexión de las segundas.

- Si históricamente ha habido una falta de interés por una enseñanza reglada del profesionalismo, la situación ha cambiado en la última década apareciendo facultades plenamente comprometidas con la orientación a los valores y el desarrollo del profesionalismo.

- La formación en profesionalismo debe atender los tres ámbitos de toda formación: contenidos o bases cognitivas, pedagogías o estrategias para enseñar y aprender, y evaluación para saber hasta qué punto los resultados obtenidos se acercan a los objetivos deseados.

\section{Bibliografía}

1. Swick HM. Toward a normative definition of medical professionalism. Acad Med 2000; 75; 612-6.

2. Blumenthal D. The vital role of professionalism in health reform. Health Affairs 1994; 13: 352-6.

3. Cruess SR, Cruess RL. Professionalism must be taught. Br Med J 1997; 315: 1674-7.

4. Hensel WA, Dickey NW. Teaching professionalism: passing the torch. Acad Med 1998; 13: 865-70.

5. Relman AS. Education to defend professional values in the new corporate age. Acad Med 1998; 73: 1229-33.

6. Wynia MK, Latham SR, Kao AC, Berg JW, Emmanuel LL Medical professionalism in society. N Engl J Med 1999; 341 $1612-6$.

7. Barondess JA. Medicine and professionalism. Arch Intern Med 2003; 163: 145-9.

8. Borrell F, Epstein R, Pardell A. Profesionalidad y profesionalismo: fundamentos, contenidos, praxis y docencia. Med Clin (Barc) 2006; 127: 337-42.

9. Cohen JJ. Linking professionalism to humanism: what it means, why it matters. Acad Med 2007; 82: 1029-32.

10. Irvine D. Professionalism: dead or alive? Lancet 2004; 364: 1479-80.

11. Levenson R, Dewar S, Shepherd S. Understanding doctors: harnessing professionalism. London: Kings Fund/Royal
College of Physicians; 2008. URL: http://www.kingsfund. org.uk/publications.

12. Oriol-Bosch A, Pardell H, Gual A. Ser médico hoy: retos del nuevo profesionalismo. Fundación Educación Médica; 2006. URL: http://www.cgcm.es/sites/default/files.

13. Pardell $\mathrm{H}$, ed. El médico del futuro. Barcelona: Fundación Educación Médica; 2009.

14. Smith AC, Kleinman S. Managing emotions in medical school: student's contacts with the living and the dead. Soc Psychol Q 1989; 52: 56-69.

15. General Medical Council. Good Medical Practice. 4 ed. GMC; 2006. URL: http://www.gmc-uk.org/static/documents/ content/GMC_GMP_0911.pdf .

16. General Medical Council. Tomorrow's Doctors. URL: http:// www.gmc-uk.org/education/documents/gmc_td_2009.pdf.

17. Accreditation Council for Graduate Medical Education. Outcome Project: general competencies. Chicago: ACGME; 2007. URL: http://www.acgme.org/outcome/comp/compFull.asp.

18. ABIM Foundation, ACO-ASIM Foundation, European Federation of Internal Medicine. La profesión médica en el nuevo milenio: estatutos para la regulación de la práctica médica. Med Clin (Barc) 2002; 118: 704-6.

19. Association of American Colleges of Medicine. Learning objectives for medical student education: guidelines for medical schools. AAMC; 1998

20. Royal College of Physicians and Surgeons of Canada. Can Meds 2005 framework. URL: http://www.rcpsf.edu/canmeds.

21. Association of Faculties of Medicine of Canada. The future of medical education in Canada: a collective vision for MD education. FMC; 2009. URL: http://www.afmc.ca/fmec.

22. Rodríguez-Sendín JJ. Definición de 'profesión médica', 'profesional médico/a' y 'profesionalismo médico.' Educ Med 2010; 13: 63-6.

23. Holtman MC. A theoretical sketch of medical professionalism as a normative complex. Adv Health Sci Educ 2008; 13: 233-45.

24. Stern D, Papadakis M. The developing physician -becoming a professional. N Engl J Med 2006; 355: 1794-9.

25. Arnold L. Assessing professional behavior: yesterday, today and tomorrow. Acad Med 2002; 77: 502-15.

26. Self DJ, Schrader DE, Balwin DC Jr, Wolinsky FD. A pilot study of the relationship of medical education and moral development. Acad Med 1991; 66: 629-5.

27. Self DJ, Olivares M, Baldwin DC Jr. Clarifying the relationship of medical education and moral development. Acad Med 1998; 73: 517-20.

28. Ginsburg S, Regher G, Lingard L. To be or not to be: the paradox of the emerging professional stance. Med Educ 2003; 37: 350-7.

29. Paice E, Heard S, Moss F. How important are role models in making good doctors? Br Med J 2002; 325: 707-10.

30. Zey-Ferrell M, Ferrell OC. Role-set configuration and opportunity as predictors of unethical behaviour in organizations. Hum Relat 1982; 35: 587-604.

31. Feudtner C, Christakis DA, Christakis NA. Do clinical clerks suffer ethical erosion? Students' perceptions of their ethical environment and personal development. Acad Med 1994; 69: 670-9.

32. Hoff TJ, Pohl H, Bartfield J. Creating a learning environment to produce competent residents: the roles of culture and context. Acad Med 2004; 79: 532-40.

33. Brass DL, Butterfield KD, Skaggs BC. Relationships and unethical behaviour: a social network perspective. Acad Manage Rev 1998; 23: 14-31.

34. Leape LL, Fromson JA. Problem doctors: is there a system level solution? Ann Intern Med 2006; 144: 107-15.

35. Hojat M, Gonella JS, Nasca TJ, Mangione S, Vergare M, Magee M. Physician empathy: definition, components, measurement, and relationship to gender and specialty. Am J Psychiatry 2002; 159: 1563-79.

36. Reiser SJ. The moral order of the medical school. In Wear D Bickel J, eds. Educating for professionalism: creating a culture of humanism in medical education. Iowa: University of Iowa Press; 2000. p. 3-10. 
37. Hafferty FC. In search of the lost cord: professionalism and the medical education's hidden curriculum. In Wear D, Bicke J, eds. Educating for professionalism: creating a culture of humanism in medical education. Iowa: University of Iowa Press; 2000. p. 11-34.

38. Swick HM, Simpson DE. Letters to the editor: another professional skills course worth nothing. Acad Med 1998; 73: 725 .

39. Craig Brater D. Infusing professionalism into a school of medicine: perspectives from the dean. Acad Med 2007; 82: 1094-7.

40. Cruess RL, Cruess SR, Steinert S. Teaching medical professionalism. New York: Cambridge University Press; 2009.

41. Brown JS, Collins A, Duguid S. Situated cognition and the culture of learning. Educ Res 1989; 18: 32-42.

42. Cruess RL, Cruess SR. Teaching professionalism: general principles. Med Teach 2006; 28: 205-8.

43. McLelland H. Situational learning perspectives. Englewood Cliffs, NJ: Educational Technology Publications; 1996.

44. Mann K. Learning and teaching in professional character development. In Kenney N, ed. Lost virtue: professional character development in medical education. New York: Elsevier; 2006.
45. Schön DA. The reflective practitioner: how professionals think in action. New York: Basic Books; 1983.

46. Wright SM, Carrese JA. Which values do attending physicians try to pass on to house officers. Med Educ 2001; 35: 941-5.

47. Kenny NP, Mann KV, McLeod H. Role modeling in physicians' professional formation: reconsidering an essential but untapped educational strategy. Acad Med 2003; 78: 1203-10.

48. Boenink AD, De Jonge P, Smal K. Oderrwald A, Van Tilburg W. The effects of teaching medical professionalism by means of vignettes: an exploratory study. Med Teach 2005; 27: 420-32.

49. Gordon J. Assessing students' personal and professional development using portfolios and interviews. Med Educ 2003; 37: 335-40.

50. Winter RO, Birnberg BA. Teaching professionalism artfully. Fam Med 2006; 38: 169-71.

51. Coulehan J. Today's professionalism: engaging the mind but not the heart. Acad Med 2005; 80: 892-8.

52. Wear D, Kucsewski MG. The professionalism movement: can we pause? Am J Bioeth 2004; 4: 1-10.

53. Hafferty FW, Franks R. The hidden curriculum, ethics teaching, and the structure of medical education. Acad Med 2006; 69: 861-71.

54. Stern DT. A framework for measuring professionalism. In Stern DT, ed. Measuring medical professionalism. Oxford: Oxford University Press; 2006. p. 3-13. 